This is the Table of Contents for the most recent online-only supplemental issues TOMM, 14(1s) and TOMM, 14(2s). Please find these supplemental issues in the ACM Digital Library and enjoy reading them!

\title{
Table of Contents: Online Supplement Volume 14, Number 1s
}

\section{Special Section on Representation, Analysis and Recognition of 3D Humans}

Introduction to the Special Issue on Representation, Analysis, and Recognition of 3D Humans Stefano Berretti, Mohamed Daoudi, Pavan Turaga, Anup Basu

Article 15

DOI: https://doi.org/10.1145/3181709

Representation, Analysis, and Recognition of 3D Humans: A Survey

Stefano Berretti, Mohamed Daoudi, Pavan Turaga, Anup Basu

Article 16

DOI: https://doi.org/10.1145/3182179

Deformation-Based 3D Facial Expression Representation

Girum G. Demisse, Djamila Aouada, Björn Ottersten

Article 17

DOI: https://doi.org/10.1145/3176649

Texture and Geometry Scattering Representation-Based Facial Expression Recognition in 2D+3D Videos

Yongqiang Yao, Di Huang, Xudong Yang, Yunhong Wang, Liming Chen

Article 18

DOI: https://doi.org/10.1145/3131345

Combining Facial Parts For Learning Gender, Ethnicity, and Emotional State Based on RGB-D Information

Safaa Azzakhnini, Lahoucine Ballihi, Driss Aboutajdine

Article 19

DOI: https://doi.org/10.1145/3152125

Early Recognition of 3D Human Actions

Sheng Li, Kang Li, Yun Fu

Article 20

DOI: https://doi.org/10.1145/3131344

A Unified Framework for Multi-Modal Isolated Gesture Recognition

Jiali Duan, Jun Wan, Shuai Zhou, Xiaoyuan Guo, Stan Z. Li

Article 21

DOI: https://doi.org/10.1145/3131343

2018 Copyright is held by the owner/author(s).

1551-6857/2018/05-ART42

https://doi.org/10.1145/3226039 
Gait Recognition from Motion Capture Data

Michal Balazia, Petr Sojka

Article 22

DOI: https://doi.org/10.1145/3152124

Sequential Articulated Motion Reconstruction from a Monocular Image Sequence

Yong Su, Zhiyong Feng, Jianhai Zhang, Weilong Peng, Meng Xing

Article 23

DOI: https://doi.org/10.1145/3180420

Full 3D Reconstruction of Non-Rigidly Deforming Objects

Hassan Afzal, Djamila Aouada, Bruno Mirbach, Björn Ottersten

Article 24

DOI: https://doi.org/10.1145/3177756

\section{Special Section on Multimedia Computing and Applications of Socio-Affective Behaviors} in the Wild

Introduction to the Special Section on Multimedia Computing and Applications of Socio-Affective Behaviors in the Wild

Fabien Ringeval, Björn Schuller, Michel Valstar, Jonathan Gratch, Roddy Cowie, Maja Pantic

Article 25

DOI: https://doi.org/10.1145/3181711

Detection of Human, Legitimate Bot, and Malicious Bot in Online Social Networks

Based on Wavelets

Sylvio Barbon Jr, Gabriel F. C. Campos, Gabriel M. Tavares, Rodrigo A. Igawa, Mario L. Proença Jr, Rodrigo Capobianco Guido

Article 26

DOI: https://doi.org/10.1145/3183506

Spatially Coherent Feature Learning for Pose-Invariant Facial Expression Recognition

Feifei Zhang, Qirong Mao, Xiangjun Shen, Yongzhao Zhan, Ming Dong

Article 27

DOI: https://doi.org/10.1145/3176646

Table of Contents: Online Supplement Volume 14, Number $2 \mathrm{~s}$

\section{Special Section on QoE Management for Multimedia Services}

Guest Editorial: Special Issue on "QoE Management for Multimedia Services"

Lea Skorin-Kapov, Martín Varela, Tobias Hoßfeld, Kuan-Ta Chen

Article 28

DOI: https://doi.org/10.1145/3192332

A Survey of Emerging Concepts and Challenges for QoE Management of Multimedia Services Lea Skorin-Kapov, Martín Varela, Tobias Hoßfeld, Kuan-Ta Chen

Article 29

DOI: https://doi.org/10.1145/3176648 
Measuring Individual Video QoE: A Survey, and Proposal for Future Directions Using Social Media Yi Zhu, Sharath Chandra Guntuku, Weisi Lin, Gheorghita Ghinea, Judith A. Redi Article 30

DOI: https://doi.org/10.1145/3183512

Quality of Experience-Centric Management of Adaptive Video Streaming Services:

Status and Challenges

Stefano Petrangeli, Jeroen van der Hooft, Tim Wauters, Filip De Turck

Article 31

DOI: https://doi.org/10.1145/3165266

SABR: Network-Assisted Content Distribution for QoE-Driven ABR Video Streaming Divyashri Bhat, Amr Rizk, Michael Zink, Ralf Steinmetz

Article 32

DOI: https://doi.org/10.1145/3183516

A Generic Approach to Video Buffer Modeling Using Discrete-Time Analysis

Valentin Burger, Thomas Zinner, Lam Dinh-Xuan, Florian Wamser, Phuoc Tran-Gia

Article 33

DOI: https://doi.org/10.1145/3183511

Can You See What I See? Quality-of-Experience Measurements of Mobile Live Video Broadcasting Matti Siekkinen, Teemu Kämäräinen, Leonardo Favario, Enrico Masala

Article 34

DOI: https://doi.org/10.1145/3165279

PMS: A Novel Scale-Adaptive and Quality-Adaptive Hybrid P2P/Multisource Solution for Live Streaming

Joachim Bruneau-Queyreix, Jordi Mongay Batalla, Mathias Lacaud, Daniel Negru

Article 35

DOI: https://doi.org/10.1145/3183515

QoE-Aware OTT-ISP Collaboration in Service Management: Architecture and Approaches

Alessandro Floris, Arslan Ahmad, Luigi Atzori

Article 36

DOI: https://doi.org/10.1145/3183517

\section{Special Section on Multimedia Understanding via Multimodal Analytics}

Guest Editorial: Special Section on "Multimedia Understanding via Multimodal Analytics"

Yan Yan, Liqiang Nie, Rita Cucchiara

Article 37

DOI: https://doi.org/10.1145/3192334

Multimodal Multiplatform Social Media Event Summarization

Akanksha Tiwari, Christian von der Weth, Mohan S. Kankanhalli

Article 38

DOI: https://doi.org/10.1145/3115433 
Structure-Aware Multimodal Feature Fusion for RGB-D Scene Classification and Beyond Anran Wang, Jianfei Cai, Jiwen Lu, Tat-Jen Cham

Article 39

DOI: https://doi.org/10.1145/3115932

Image Captioning with Deep Bidirectional LSTMs and Multi-Task Learning Cheng Wang, Haojin Yang, Christoph Meinel

Article 40

DOI: https://doi.org/10.1145/3115432

Toward Personalized Activity Level Prediction in Community Question Answering Websites Zhenguang Liu, Yingjie Xia, Qi Liu, Qinming He, Chao Zhang, Roger Zimmermann Article 41

DOI: https://doi.org/10.1145/3187011 